\title{
Optimizing the Number of Double-Differenced Observations for GPS Networks in Support of Deformation Monitoring Applications
}

\author{
Volker Janssen \\ School of Geomatic Engineering \\ The University of New South Wales \\ Sydney NSW 2052 AUSTRALIA
}

\begin{abstract}
Deformation monitoring using GPS is usually carried out by installing and operating a local network of GPS receivers mounted on the deforming body, e.g. the flanks of a volcano. For continuous monitoring applications a near-realtime, epoch-by-epoch solution obtained from multibaseline processing is desired in order to take into account between-baseline correlations and to detect movements over as short a period of time as possible. In the case of the volcano monitoring application, the sides of the volcano will block out part of the sky, hence the receivers are not likely to track a lot of satellites that are visible from all receiver stations at the same time. If the usual base-station / base-satellite approach is used in the baseline processing, only the common satellites are considered, resulting in the number of possible double-differenced observables being comparatively low, hence a lot of valuable information may be lost. The proposed method based on the work by Saalfeld (1999) considers satellites that are visible from a small number of network stations only. Hence the number of independent double-differenced observables can be maximised in order to obtain a better solution. A numerical example is given which verifies the improved solutions that can be obtained using this data processing approach.
\end{abstract}

\section{INTRODUCTION}

Deformation monitoring using GPS is usually carried out by installing and operating a local network of GPS receivers located on and around the deforming body, e.g. the flanks of a volcano, or on a dam wall, bridge structure, etc. Multibaseline processing strategies should be used because all baselines are then computed together, taking into account the between-baseline correlations which arise from observing a network simultaneously. Commercial software packages use a baseline-by-baseline approach, processing one baseline after the other, treating the baselines as independent measurements and therefore neglecting these correlations. For continuous deformation monitoring applications, a near-realtime, epoch-by-epoch solution is desired in order to detect movements over a short period of time.

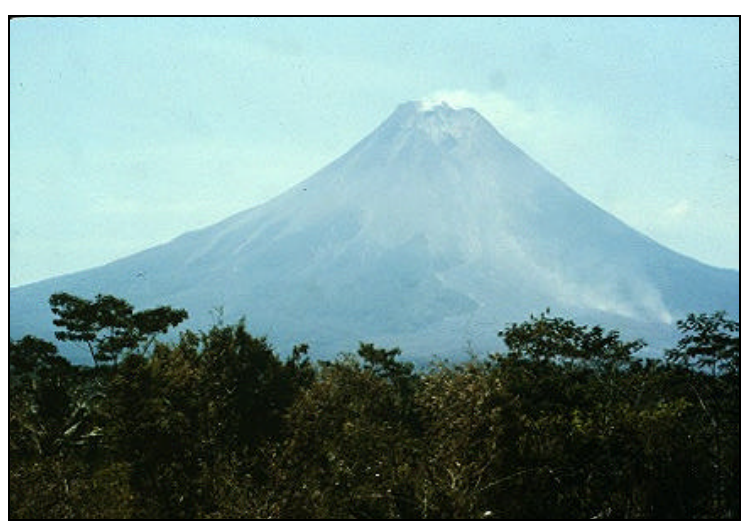

Fig. 1: Gunung Merapi, Indonesia

In the case of the volcano monitoring application, the sides of the volcano will block out part of the sky, hence the receivers are not likely to track a lot of satellites that are visible from all receivers at the same time. Figure 1 shows the typical situation around a volcano. If the usual base-station / basesatellite approach is used in the data processing, only the common satellites are considered, resulting in the number of possible doubledifferences being comparatively low, hence a lot of valuable information can be lost. In the worst case, a solution may not be obtained if the number of common satellites tracked by all network stations is reduced to less than four. The proposed method considers satellites that are visible from a small number of network stations only. Hence, the number of independent double-differenced observables can be maximised in order to generate a more accurate and reliable solution.

\section{GENERATING AN INDEPENDENT SET OF DOUBLE-DIFFERENCES}

When processing a local GPS network, doubledifferenced observables between two receivers and two satellites are used, rather than raw carrier 
phase measurements, because the receiver and satellite clock errors are eliminated by differencing. The double-difference between two receivers $m$ and $\mathrm{n}$ and two satellites $\mathrm{i}$ and $\mathrm{j}$ can be obtained by (e.g. Hofmann-Wellenhof et al., 1997; Rizos, 1997):

$$
\Phi_{\mathrm{mn}}^{\mathrm{ij}}=\Phi_{\mathrm{n}}^{\mathrm{j}}-\Phi_{\mathrm{n}}^{\mathrm{i}}-\Phi_{\mathrm{m}}^{\mathrm{j}}+\Phi_{\mathrm{m}}^{\mathrm{i}}
$$

where $\Phi_{\mathrm{m}}^{\mathrm{i}}$ denotes the carrier phase measurement between receiver $m$ and satellite $i$. Usually the common satellites visible from all network GPS stations are determined and a "base-station" and a "base-satellite" are selected to generate these double-differenced observables. The proposed data processing approach determines the receiver-tosatellite connections for each site of the network. A maximum set of independent double-differenced combinations is computed using vector space methods, and the geometric characterisations of Boolean matrices, as suggested by Saalfeld (1999).

In this algorithm a Boolean matrix of 0 s and $1 \mathrm{~s}$ represents the receiver-to-satellite connections, where a 1 denotes that a certain satellite has been received by a certain network site. If this matrix is built so that the receivers are arranged in rows and the satellites are arranged in columns, an axisparallel rectangle of $1 \mathrm{~s}$ will represent a possible double-difference (Fig. 2).

\begin{tabular}{|c|c|c|c|c|c|c|c|}
\hline & $\mathrm{S}_{1}$ & $\mathrm{~S}_{2}$ & $\mathrm{~S}_{3}$ & $\mathrm{~S}_{4}$ & $\mathrm{~S}_{5}$ & $\mathrm{~S}_{6}$ & $\mathrm{~S}_{7}$ \\
\hline $\mathrm{R}_{1}$ & 1 & 0 & 1 & 1 & 0 & 1 & 1 \\
\hline $\mathrm{R}_{2}$ & 1 & 1 & 1 & 1 & 0 & 1 & 1 \\
\hline $\mathrm{R}_{3}$ & 1 & 1 & 1 & 0 & 1 & 1 & 0 \\
\hline $\mathrm{R}_{4}$ & 1 & 0 & 1 & 1 & 1 & 0 & 1 \\
\hline
\end{tabular}

Fig. 2: Receiver-to-satellite connections for 4 receivers and 7 satellites

Some of these rectangles can be formed by appending or subtracting two other rectangles that share a common side. These newly formed rectangles correspond to linearly dependent double-differences. The matrix is processed by rows, and within each row by columns. For each element containing a 1 , the submatrix having this element in the lower-right corner is formed. By rearranging the rows and columns of the submatrix and reducing its size, the set of independent double-differences is obtained. Note that this set no longer refers to just one base-station and a certain base-satellite. A detailed description of the algorithm can be found in Saalfeld (1999).

\section{OPTIMISING THE DOUBLE- DIFFERENCING OPERATOR}

The set of independent double-differences is used to build up the optimised double-differencing operator that transforms the raw phase measurements into independent double-differenced observables. This double-differencing operator will take into account all available information, i.e. the additional double-differenced observables, resulting in a stronger solution. If $\mathrm{D}$ denotes the double-differencing operator and $\Phi$ denotes the vector of one-way (satellite-to-receiver) carrier phase measurements, the vector of doubledifferences $\Phi_{\mathrm{D}}$ can be obtained by:

$$
\Phi_{\mathrm{D}}=\mathrm{D} \cdot \Phi
$$

Using a double-differencing operator is crucial in the multi-baseline processing mode so as to account for the between-baseline correlations introduced by simultaneously observing all the GPS network stations (Craymer et al., 1992).

\section{AMBIGUITY RESOLUTION}

Very high accuracy initial coordinates $( \pm 5 \mathrm{~cm})$ are used in order to resolve the carrier phase ambiguities on an epoch-by-epoch basis. The initial coordinates are obtained from the previous epoch's solution and introduced as observations in order to obtain a non-singular normal matrix. This procedure assumes that the deformation around the volcano takes place gradually, without any "jumps" like those experienced during crustal deformation caused by earthquakes. Note that cycle slips do not have to be considered in an epoch-by-epoch solution such as this.

\section{NUMERICAL EXAMPLE}

Data collected from a network of six GPS receivers in Sydney, Australia, with baseline lengths ranging from $2.0 \mathrm{~km}$ to $2.7 \mathrm{~km}$ were used to test the new data processing procedure. Figure 3 is a sketch of the network. A 2-hour session was observed on the 12th October 1999 using Leica CRS1000 receivers, equipped with choke ring antennas to minimise the effect of multipath. The sampling rate was set to 30 seconds, with a satellite elevation mask of $15^{\circ}$. 


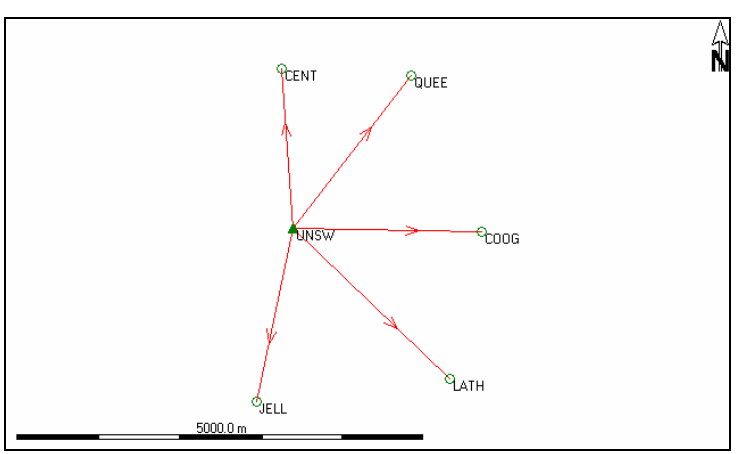

Fig. 3: GPS Network

The coordinate results obtained from the processing of the complete 2-hour session were used as "true" coordinates. The data set was then altered by blocking out part of the sky at the network stations. At each site a different azimuth band of $40^{\circ}$ pointing towards the centre of the network, with satellite elevations of less than $50^{\circ}$, was blocked out in order to simulate the situation around a volcano. The results of the conventional epoch-by-epoch solution and the proposed approach were compared.

Using the proposed approach, the number of double-differenced observables at each epoch was increased by an average of 9.8, with a maximum difference between the approaches of 20 observations (Fig. 4). The minimum number of independent double-differenced observables required to obtain a solution for a network of six sites is 15 . For certain epochs no solution could be obtained using the conventional approach because the number of common satellites received by all network stations dropped to three, resulting in only 10 double-differenced observables. However, using the proposed method satisfactory solutions could be obtained.

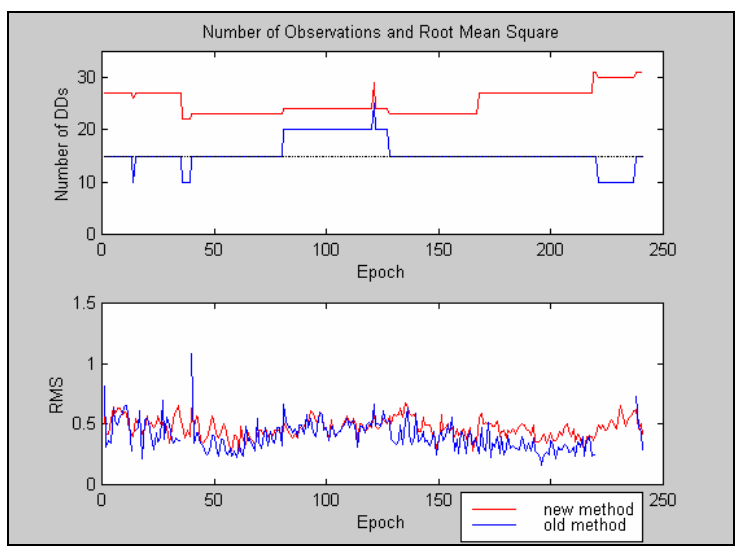

Fig. 4: Number of Observations and Root Mean Square
Figure 4 also shows that the root mean square of the double-differenced residuals was improved. The standard deviation of the root mean square was reduced by $34 \%$, from 0.1232 to 0.0811 .

The coordinates obtained by both data processing approaches were compared against the "true" values. Figures 5a-5e show the resulting coordinate differences. Note that the gaps in the curve representing the conventional approach are caused by an insufficient number of doubledifferenced observables for those epochs. It is evident that the proposed method increases the quality of the results. The coordinate variations obtained by using the proposed method are also smaller and smoother. This can be verified from the standard deviations of the $\mathrm{X}-, \mathrm{Y}-$ and $\mathrm{Z}$ components (Figures 6a-6e).

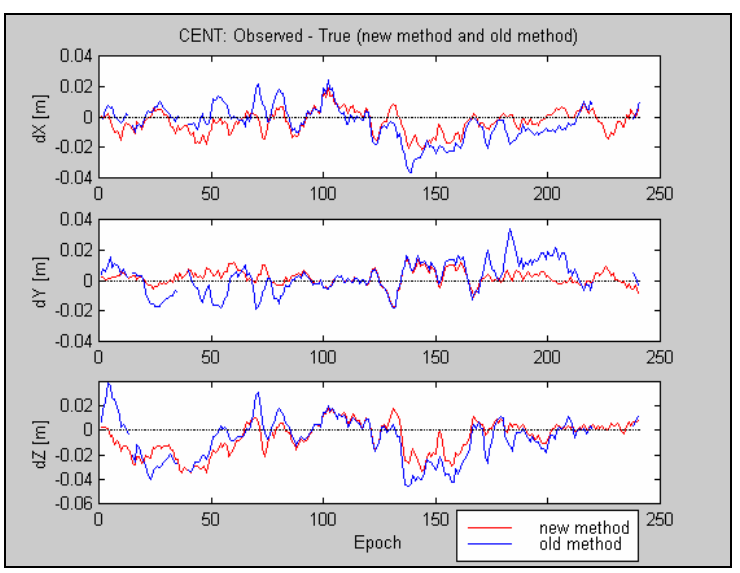

Fig. 5a: Obtained coordinates compared to 'truth' (CENT)

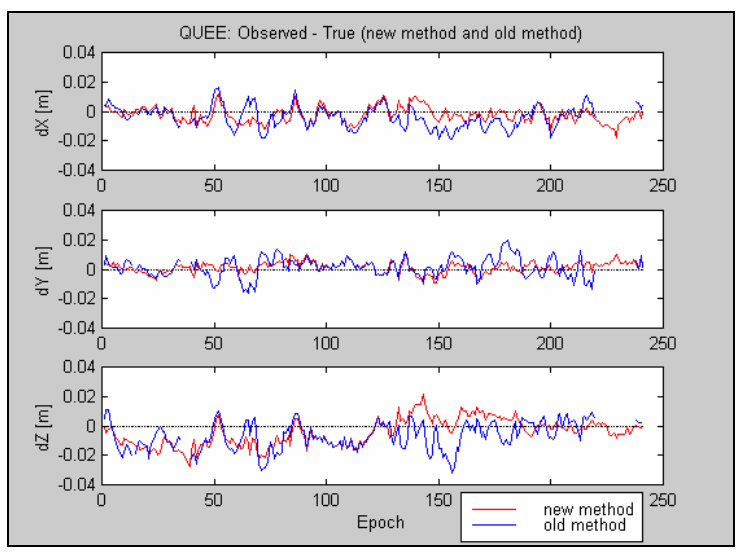

Fig. 5b: Obtained coordinates compared to 'truth' (QUEE) 


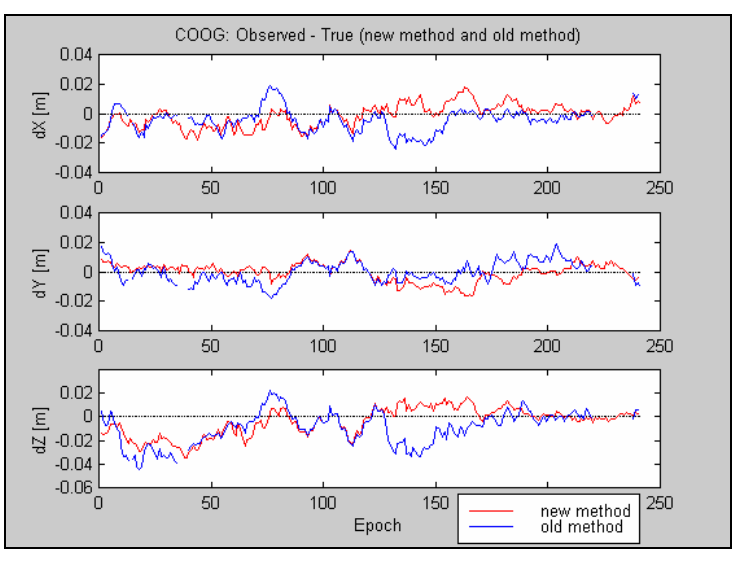

Fig. 5c: Obtained coordinates compared to 'truth' (COOG)

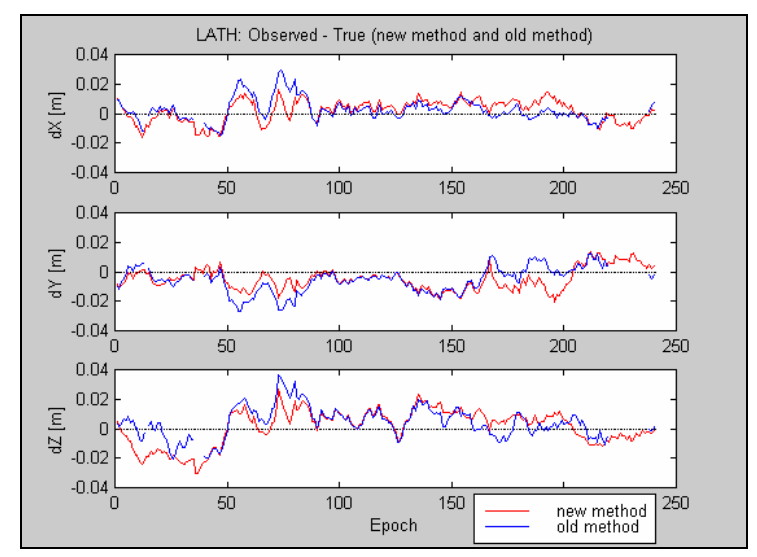

Fig. 5d: Obtained coordinates compared to 'truth' (LATH)

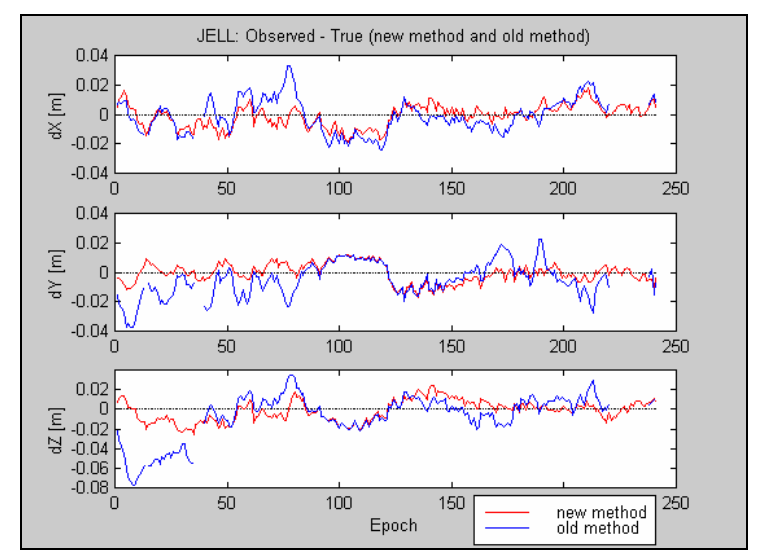

Fig. 5e: Obtained coordinates compared to ‘truth' (JELL)

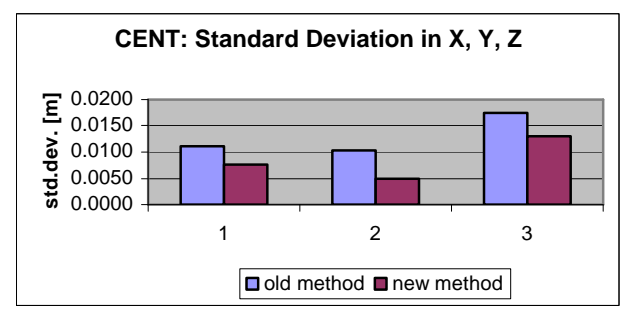

Fig. 6a: Standard deviations (CENT)

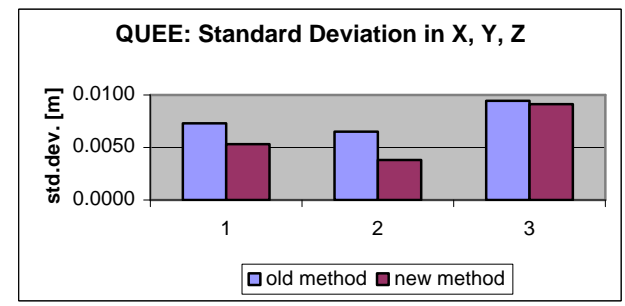

Fig. 6b: Standard deviations (QUEE)

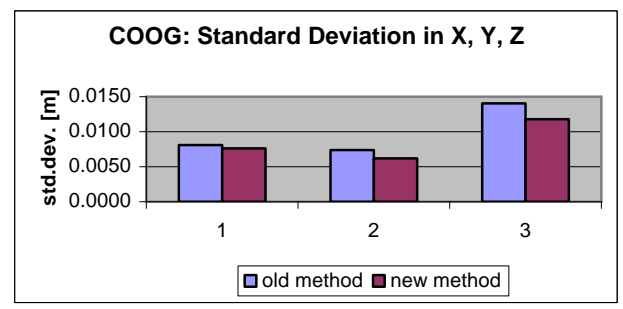

Fig. 6c: Standard deviations (COOG)

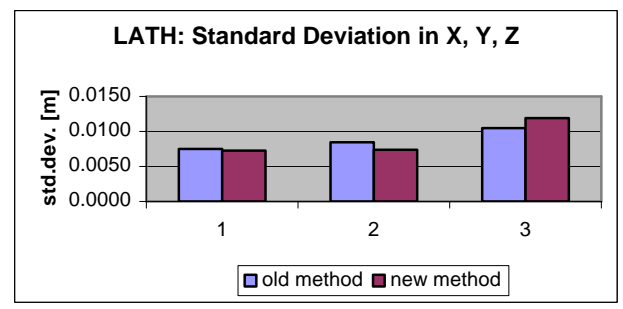

Fig. 6d: Standard deviations (LATH)

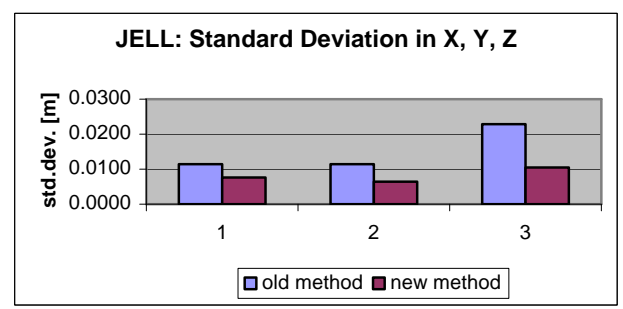

Fig. 6e: Standard deviations (JELL)

\section{CONCLUDING REMARKS}

The numerical example has demonstrated that the proposed data processing methodology does indeed improve the coordinate results. The number of double-differenced observables available to compute a solution is maximised by using all available information, therefore optimising the quality of the results. A solution can be obtained even if less than four common satellites can be received simultaneously at all network stations. Hence the problem of the deforming body obstructing part of the sky, and thereby reducing the effectiveness of certain deformation monitoring 
applications, can be overcome using the proposed data processing approach.

\section{ACKNOWLEDGEMENTS}

The author is supported by funding from the Australian Research Council and by an Overseas Postgraduate Research Scholarship (OPRS). The assistance of members of the SNAP group (Satellite Navigation And Positioning) at The University of New South Wales in collecting the data is gratefully acknowledged. My supervisors A/Prof. Chris Rizos and Dr. Shaowei Han are thanked for their valuable suggestions.

\section{REFERENCES}

Craymer, M.R., and N. Beck (1992): Session Versus Single-Baseline GPS Processing, Proceedings of ION-GPS-92, Albuquerque, New Mexico, 16-18 September, 995-1004.

Hofmann-Wellenhof, B., H. Lichtenegger and J. Collins (1997): GPS Theory and Practice, $4^{\text {th }}$ edition, Springer, Wien, Austria, 389 pp.

Rizos, C. (1997): Principles and Practice of GPS Surveying, Monograph 17, School of Geomatic
Engineering, The University of New South Wales, Sydney, Australia, 555 pp.

Saalfeld, A. (1999): Generating Basis Sets of Double Differences, Journal of Geodesy, 73, 291297.

\section{BIOGRAPHY}

Volker Janssen graduated with a Dipl.-Ing. from the University of Bonn, Germany, in 1997. He is curently a Ph.D. student in the SNAP (Satellite Navigation And Positioning) group at The University of New South Wales, Sydney, Australia, working in the field of volcano deformation monitoring with GPS.

\section{Correspondence}

Volker Janssen

School of Geomatic Engineering

The University of New South Wales

Sydney NSW 2052, AUSTRALIA

Ph: +61-2-9385 4208

Fax: +61-2-9313 7493

Email: v.janssen@student.unsw.edu.au 